\title{
Ethnologies
}

Harald Welzer, Sabine Moller et Karoline Tschuggnall.

"Grand-Père n'était pas un nazi ". National-socialisme et Shoah dans la mémoire familiale. (Paris, Gallimard, 2013. Pp. 344. Coll. « NRF essais ». ISBN 978-2-07-013589-9)

\section{Adrien Genoudet}

Volume 35, numéro 2, 2013

URI : https://id.erudit.org/iderudit/1026557ar

DOI : https://doi.org/10.7202/1026557ar

Aller au sommaire du numéro

Éditeur(s)

Association Canadienne d'Ethnologie et de Folklore

ISSN

1481-5974 (imprimé)

1708-0401 (numérique)

Découvrir la revue

Citer ce compte rendu

Genoudet, A. (2013). Compte rendu de [Harald Welzer, Sabine Moller et Karoline Tschuggnall. « Grand-Père n'était pas un nazi ». National-socialisme et Shoah dans la mémoire familiale. (Paris, Gallimard, 2013. Pp. 344. Coll. « NRF essais ». ISBN 978-2-07-013589-9)]. Ethnologies, 35(2), 207-211.

https://doi.org/10.7202/1026557ar d'utilisation que vous pouvez consulter en ligne. 
though, is appealing to any lover of folktales. The book might be most useful for students of Slavic culture and language.

Lydia Bringerud

Memorial University

Harald Welzer, Sabine Moller et Karoline Tschuggnall. "Grand-Père n'était pas un nazi ". National-socialisme et Shoah dans la mémoire familiale. (Paris, Gallimard, 2013. Pp. 344. Coll. "NRF essais". ISBN 978-2-07-013589-9)

La sortie récente de la traduction française de l'ouvrage intitulé «Grand-Père n'était pas un nazi ». National-socialisme et Shoah dans la mémoire familiale de Harald Welzer, Sabine Moller et Karoline Tschuggnall, traduit par Olivier Mannoni, a permis d'attirer l'attention sur la manière dont les différentes générations allemandes ont reçu et décanté le passé nationalsocialiste et la Shoah. L'ouvrage a paru, au départ, aux Éditions Fisher à Francfort en 2002. C'est donc avec un certain recul que nous recevons cette longue enquête-analyse réalisée en Allemagne pendant plusieurs années, qui a recueilli les témoignages de 40 familles et de 182 entrevues individuelles ou entretiens familiaux (313). Harald Welzer est psychosociologue et dirige à Essen le Centre de recherche interdisciplinaire sur la mémoire auquel collabore Sabine Moller qui est, quant à elle, à l'Université Humboldt de Berlin. Karoline Tschuggnall est membre de l'Institut de psychologie de l'Université libre de Berlin.

Autant dire que l'on décèle aisément l'axe « histoire psychologique » à la lecture de ce livre qui permet ainsi, au fil des entretiens et de leurs analyses, de comprendre que la "mémoire »du Troisième Reich est extrêmement lacunaire - voire faussée - au sein des familles. Une lacune qui semble s'intensifier au fil des générations.

Pour démontrer ce constat annoncé dès l' « avertissement aux lecteurs » (7) et pour l'illustrer, les auteurs se servent de ces dizaines d'entretiens et s'appuient sur un certain nombre de citations extraites directement 
de ces enregistrements. On peut voir ainsi comment le langage permet la construction d'une mémoire familiale (22) et comment les histoires racontées sont entendues puis déformées ou réinterprétées au fil des années (57). La forme analytique du livre oscille donc entre ce matériau brut (les entretiens) et leurs commentaires par les auteurs. Se déploie dès lors une réflexion sur la construction du souvenir, de la mémoire et des processus de transmission au sein des familles et au fil des générations jusque dans les ambivalences géographiques marquées par la scission entre la RDA et la RFA (220).

Les lecteurs peuvent se référer à quelques tableaux en fin d'ouvrage (291) qui résument l'échantillon analysé et qui permettent une vue plus générale et davantage statistique sur le contenu. Enfin, pour appuyer leurs enquêtes et leurs analyses, conscients que les " résultats sur la transmission sont généralisables, mais pas représentatifs » (287), les auteurs ont fait réaliser un sondage représentatif en juin 2002 par l'Institut Emnid à Bielefeld, sondage qui corrobore la majorité des conclusions avancées et qui est présenté dans la « Postface à la seconde édition » en fin d'ouvrage (285). Au-delà d'un possible traitement trop «psychologique » et d'une possible volonté de trop appuyer un pressentiment transformé en thèse, il semble intéressant de nous concentrer sur le cinquième chapitre en particulier, parce qu'il soulève de nombreuses questions - à la fois historiques, méthodologiques et culturelles.

Le cinquième chapitre, "Le général du diable". Le cinéma comme biographie ou Comment les médias façonnent le souvenir » (142), pose la question passionnante, à travers certains entretiens, de la place de l'image dans la construction et la transmission des souvenirs. Les auteurs ont remarqué au fil des entrevues que les témoins faisaient souvent référence à un film, à des actualités filmées ou à une image pour appuyer leurs discours. Ils ont donc décidé de regrouper ces éléments constitutifs de la "mémoire visuelle » des personnes sollicitées et tentent une analyse plus large sur la manière dont cette mémoire visuelle conditionne la transmission et l'appropriation des souvenirs par les jeunes générations (146).

Les auteurs soulignent dans un premier temps la manière dont les images et leur diffusion massive permettent de « remplir les vides » des souvenirs et, pour les jeunes générations, d'illustrer les récits entendus (151). Ainsi, ils montrent que la plupart des jeunes générations ont construit et «brodé » sur les histoires racontées par leurs parents ou leurs grands-parents, à partir des différents éléments visuels qui composent leur culture. De plus, les récits racontés et entendus sont de plus en plus, selon les auteurs, jugés - du point de vue de la véracité des faits racontés - à partir de la propre culture 
visuelle des personnes qui reçoivent le discours. Ils notent : « [l]'exactitude et la plausibilité des récits sont ici, de plus en plus, mesurés à l'aune de leur capacité à s'accorder avec l'inventaire visuel que les médias ont mis à disposition » (147). Le deuxième aspect sur lequel les auteurs insistent longuement est la manière dont les témoins utilisent les films de fiction pour raconter des événements qu'ils ne souhaitent pas ou n'arrivent pas à raconter. Ils mettent en avant Mathilde Beck, fille d'un membre des SA qui s'est suicidé en 1940. On comprend, au fil de son entretien et dans la manière dont il est restitué par les auteurs, qu'elle s'appuie sur le film de Helmut Kautner, Le général du diable, de 1955, où il est question d'un général de la Wehrmacht qui se suicide pour sauver son honneur. En s'appuyant sur ce film, les auteurs montrent que Mme Beck reconstruit la disparition brutale de son père et lui donne du sens. Mais ils montrent également comment cet appui sur l'image a conditionné, au fil des générations, la lecture de la mort du père ou du grand-père (160).

Les auteurs tentent finalement une investigation surprenante. En considérant que nombre de récits sont souvent le fruit d'appropriations diverses - et notamment issues du cinéma - ils essaient de retrouver des références cinématographiques au sein des discours. Cette démarche d'investigation semble poser problème. En effet, à aucun moment - sauf dans un seul exemple qui cite explicitement le film de Bernard Wicki (171) - les témoins ne font référence à cet héritage culturel et visuel ; et il ne leur est pas demandé s'il y font référence explicitement ou non. Ainsi, les auteurs apposent ici une réflexion a posteriori sur des discours enregistrés et se servent de leur propre savoir pour tenter de déceler de quelconques héritages ou influences culturels et visuels.

C'est tout le problème - et la critique que l'on pourrait formuler - de ce chapitre plein de promesses sur les rapports entre construction du souvenir, transmission et écriture de l'histoire par l'image (qu'elle soit individuelle ou collective) : à aucun moment - ou du moins dans les passages qui nous sont proposés - nous ne pouvons voir se dessiner une critique et une analyse de l'impact de ces images dans la construction, la modification, la déformation, l'écriture, du souvenir et de sa transmission. Seul, au milieu du chapitre, l'exemple de Paul Boesch - de la "génération des enfants »- semble effleurer cette possible réflexion (162). La culture visuelle, dans son grand ensemble, n'est-elle pas un des premiers vecteurs d'écriture et de perception des périodes historiques - et notamment pour les nouvelles générations ? N'est-ce pas, avant tout, au sein de l'analyse et de la compréhension de la culture visuelle que se dessine cette impureté - qui caractérise la perception 
imagée de l'histoire?

Un dernier élément paraît important à relever car il semble être passé inaperçu et reste peu commenté au sein de l'ouvrage. À la lecture du premier chapitre, on apprend, au détour d'une phrase, qu'un montage d'images cinématographiques a précédé les entretiens. Dans une note, on peut lire que « les entretiens familiaux étaient précédés d'un montage de dix minutes de films d'amateurs remontant aux années 1930 et 1940, qui faisait office d'introduction à la discussion thématique » (311). Cette démarche et ce dispositif ne sont à aucun moment mis en perspective au sein de l'analyse. Quelques références à cette « introduction » visuelle parsèment le commentaire, mais elles ne semblent jamais s'intéresser au dispositif et au contenu - et au choix - de ces films. Cette note nous renvoie à une double page, à la fin du livre, qui nous présente la filmographie présentée aux personnes interviewées sous forme d'un montage de dix minutes. Cet annexe est intitulé : "Treize séquences cinématographiques ayant servi d'introduction aux conversations familiales ». On trouve, dans ce descriptif, une sélection hétéroclite ne comprenant pas uniquement des « films d'amateurs » (cette dénomination n'est pas non plus explicitée) mais aussi des films de propagande nazie.

Dès lors, on perçoit les nombreux problèmes heuristiques que posent cette utilisation et cette sélection. La simple présentation de ces films, dans un montage, pour introduire un entretien sur le souvenir et la "mémoire familiale », modifie le rapport au passé que l'on va tenter d'aborder par l'entretien oral. Mêler, dans un même ensemble, des films de propagande - qui sont ici colorisés et donc peut-être pas d'origine - et des films sur la Shoah produit nécessairement des effets de considérations et de perceptions sur la période et sur l'interviewé. Ce type de montage introductif a pour conséquence l'aplanissement du régime de croyance et de vérité que l'on entretient avec ce flot d'images. Vues, ces images produisent un effet d'illustration de la période «Troisième Reich » ou «Shoah » et ce procédé nie la démarche essentielle qui consiste en la sélection et l'addition d'images qui ne sont pas de même nature.

Malheureusement, à aucun moment, la monstration et l'élaboration de ce montage ne sont interrogées ni discutées au regard des résultats des entrevues. Pourquoi ce montage ? Qui l'a réalisé et dans quels buts? Quels effets une telle projection en «introduction » a-t-elle pu avoir ? Comment penser le souvenir et sa transmission à travers le prisme de ce type de synthèse visuelle du passé ? Ces questions semblent essentielles pour comprendre ces systèmes et ces processus de transmission. Laissées en 
suspens, ces questions méthodologiques produisent une forme de malaise qui confine au doute et qui, en définitive, amènent un léger sentiment de suspicion à l'égard de certaines conclusions scientifiques apportées.

Adrien Genoudet

Université Paris VIII Vincennes-Saint-Denis Institut de l'Histoire du Temps Présent (CNRS)

Michael Newton, ed. Celts in the Americas. (Sydney, NS: 2013, Cape Breton University Press. Pp. 376. ISBN: 978-1-897009-75-8)

Celts in the Americas, edited by Michael Newton, draws together specialists on Celtic ethnolinguistic groups in the Americas to detail how Bretons, Welsh, Scottish Gaels, Irish, and Cornish immigrants created place, community identity, and cultural cohesion through spoken and written language use in diasporic settings (the Manx are not represented: no scholar could be found to discuss the history of Manx immigrants). This book - a collection of papers from an eponymous conference held at St. Francis Xavier University in 2011, plus five invited chapters and an introduction - means to redress outdated tendencies in Celtic Studies scholarship, including the perpetuation of an anglocentric master narrative that elides the "identity and historical experiences of Celtic-speaking peoples in the Americas" under those of their colonial rulers. The chapters within the book address the different Celtic-speaking peoples individually, explicitly rejecting a scholastic history of crafting an essentializing narrative for all Celts in the Americas, and for each ethnolinguistic group. The authors overall recuperate the Celtic peoples as active agents rather than passive victims of internal colonialism while also redressing their elision in academic discourse. Primarily concerned with language and "cultural expressions" tied to language (print media and literature), the book's strong disciplinary foundation uses an established methodology within Celtic Studies - textual analysis and mapping language use and retention - while also gesturing towards interdisciplinary cultural analysis. 